\title{
Biofertilizantes na produção da videira Isabel
}

\section{Biofertilizers in production of vine Isabel}

\author{
Francisca Lacerda da Silva $\mathbb{D}^{1}$, Alex Serafim de Lima $\mathbb{D}^{1} *$, Jéssica da Mota Santos $\mathbb{1}^{1}$, Jackson de \\ Mesquita Alves ${ }^{(1}{ }^{l}$, Caio da Silva Sousa ${ }^{(1}{ }^{1}$, José Geraldo Rodrigues dos Santos ${ }^{(\mathbb{D}}{ }^{2}$
}

${ }^{1}$ Graduandos em Licenciatura Plena em Ciências Agrárias, Universidade Estadual da Paraíba. Fones: (83) 996649962, (83) 998547379, (83) 996305857, (83) 996261807, (83) 998054429; e-mails: franciscalacerdadasilva@hotmail.com, motta.jessica@ymail.com, alexcdf14@gmail.com, mesquitajackson2018@gmail.com, caio silvacr@hotmail.com; ${ }^{2}$ Professor e Doutor na Universidade Estadual da Paraíba, Campus Catolé do Rocha. Fone: (83) 996086554, e-mail: josegeraldo@ccha.uepb.edu.br

\section{A R T I G O}

Recebido: $10 / 12 / 2018$

Aprovado: 20/03/2019

\section{Palavras-chave: \\ Vitis labrusca \\ Fertilizante orgânico \\ Produtividade}

Key words:

Vitis labrusca

Organic fertilizer

Productivity

\section{R E S U M O}

A videira "Isabel" é uma das culturas mais importantes tanto para o setor agrícola quanto para subsistência de pequenas famílias. O biofertilizante bovino surge como uma alternativa para substituir o uso excessivo de adubos químicos nas pequenas propriedades, em que pode ser utilizado em cultivos agrícolas proporcionando aspectos de qualidade e quantidade, agindo positivamente no sistema solo-água-planta. Nesta perspectiva, objetivou-se avaliar a produção de frutos de videira Isabel, adubadas com tipos e doses de biofertilizante bovino, durante o segundo ciclo produtivo. $\mathrm{O}$ experimento consistiu em delineamento em blocos casualisados, com fatorial $5 \times 8$, referente a cinco tipos de biofertilizante: $\left(\mathrm{B}_{1}=\right.$ à base de esterco bovino, $\mathrm{B}_{2}=\mathrm{B}_{1}+$ farinha de rocha $\mathrm{MB} 4, \mathrm{~B}_{3}=\mathrm{B}_{2}+$ leguminosa (Vigna unguiculata L. Walp.), $\mathrm{B}_{4}=\mathrm{B}_{2}+$ cinza de madeira e $\mathrm{B}_{5}=\mathrm{B}_{3}+$ cinza de madeira) e de 8 doses de biofertilizante $\left(D_{1}=0 ; D_{2}=0,35 ; D_{3}=0,7 ; D_{4}=1,05 ; D_{5}=1,4 ; D_{6}=1,75 ; D_{7}=2,1 ;\right.$ e $D_{8}=$ 2,45 L/planta/vez), aplicadas a cada 2 meses. Os tipos de biofertilizante $\mathrm{B}_{1}, \mathrm{~B}_{2}, \mathrm{~B}_{4}$ e $\mathrm{B}_{5}$ exerceram valores significativos na produção, exceto para com a qualidade. $\mathrm{O}$ uso de biofertilizante aplicado $1,2 \mathrm{~L} /$ planta/vez proporciona maior eficiência produtiva de frutos de videira Isabel nos tipos $\mathrm{B}_{1}, \mathrm{~B}_{2}, \mathrm{~B}_{4}$ e $\mathrm{B}_{5}$, exceto para com ${ }^{\circ} \mathrm{Brix}$ e acidez. A aplicação de diferentes tipos de biofertilizante bovino associado a dosagens ótimas acarreta em plantas com elevado potencial produtivo da videira Isabel. \begin{abstract}
A B S T R A C T
The "Isabel" vine is one of the most important crops both for the agricultural sector and for subsistence of small families. The bovine biofertilizer appears as an alternative to replace the excessive use of chemical fertilizers in small properties, where it can be used in agricultural crops providing quality and quantity aspects, acting positively in the soil-waterplant system. In this perspective, the objective was to evaluate the production of Isabel grape fruits, fertilized with types and doses of bovine biofertilizer, during the second productive cycle. The experiment consisted of a randomized block design, with factorial $5 \mathrm{x}$ 8 , referring to five types of biofertilizer: $\left(B_{1}=\right.$ bovine manure, $B_{2}=B_{1}+M B 4$ rock meal, $\mathrm{B}_{3}=\mathrm{B}_{2}+$ legume (Vigna unguiculate $\mathrm{L}$. Walp.), $\mathrm{B}_{4}=\mathrm{B}_{2}+$ wood ash and $\mathrm{B}_{5}=\mathrm{B}_{3}+$ wood ash) and 8 doses of biofertilizer $\left(D_{1}=0 ; D_{2}=0.35 ; D_{3}=0.7 ; D_{4}=1.05 ; D_{5}=1.4 ; D_{6}=\right.$ $1.75 ; \mathrm{D}_{7}=2.1$ and $\mathrm{D}_{8}=2.45 \mathrm{~L} /$ plant/time), applied every 2 months. The types of biofertilizer $\mathrm{B}_{1}, \mathrm{~B}_{2}, \mathrm{~B}_{4}$ and $\mathrm{B}_{5}$ exert significant values in production, except for quality. The use of biofertilizer applied 1,2 L/plant/time provides greater productive efficiency of Isabel vine fruits in types $\mathrm{B}_{1}, \mathrm{~B}_{2}, \mathrm{~B}_{4}$ and $\mathrm{B}_{5}$, except for ${ }^{\circ} \mathrm{Brix}$ and acidity. The application of different types of bovine biofertilizer associated to optimal dosages leads to plants with high productive potential.
\end{abstract}

\section{INTRODUÇÃ̃O}

A videira, pertencente à família Vitaceae, gênero Vitis, é uma das mais antigas plantas cultivadas pelo homem. Essa cultura possui grande importância econômica, a qual gera muitos empregos diretos e indiretos nos setores de insumos, processamento, serviço de apoio, produção, distribuição e

\section{Revista Verde}

ISSN 1981-8203

Pombal, Paraíba, Brasil turismo (NETO; SOUSA, 2018). Além disso, o cultivo da videira tem promovido a sustentabilidade da agricultura familiar e o desenvolvimento de algumas regiões do planeta (FERRANTI, 2017). De acordo com Kist et al. (2018) os maiores produtores mundiais de uvas são: China, Estados Unidos, França e Itália. 
No Brasil, a viticultura ocupa uma área plantada de 81 mil ha, sendo cultivada em diferentes regiões brasileiras, porém concentra-se principalmente na região Sul do país. Com destaque ao Rio Grande do Sul, maior região vitícola brasileira, contribuindo com 777 milhões de quilos de uva por ano, dos quais são produzidos anualmente, em média, 330 milhões de litros de vinhos e mostos (sumo de uvas frescas que ainda não tenham passado pelo processo de fermentação), e aproximadamente 52 milhões de litros do suco de uva integral (MAPA, 2015).

Os plantios de uva estão diretamente relacionados às produções em pequenas propriedades e grande necessidade de mão de obra, o que faz com que contribua de forma direta para a geração de empregos constantemente. Considerando todas as atividades durante à produção de uva, desde a implantação de vinhedos até o beneficiamento e comercialização do produto, a tecnologia gera o emprego de aproximadamente 1,5 pessoa ha ${ }^{-1}$ (MELLO, 2016).

A produção agrícola gera diferentes resíduos, na maioria das vezes são orgânicos e podem ser reutilizados como fertilizantes orgânicos. Surgindo como uma alternativa em potencial para substituir os fertilizantes minerais. Entre esses fertilizantes orgânicos, os biofertilizantes líquidos, produzidos a partir da fermentação da matéria orgânica com água, tanto de forma aeróbia quanto anaeróbia são bastante utilizados. Dependendo do material utilizado para a produção dos biofertilizantes, sua composição pode apresentar todos os macro e micronutrientes essenciais para crescimento e desenvolvimento das plantas (OLIVEIRA et al., 2017).

Vários estudos com biofertilizantes em diferentes culturas agrícolas mostram resultados positivos, por exemplo, Mazaro et al. (2013) avaliaram a produção e qualidade de morangos e observaram aumento na produtividade, massa média de frutos e número de frutos em função das maiores concentrações de biofertilizante. Lima et al. (2019) observaram efeitos relevantes na cultura do maracujazeiro amarelo devido ao aumento da frequência de aplicação do biofertilizante. Chiconato et al. (2013), estudando a cultura da alface, observaram que maiores doses de biofertilizante resultaram em plantas mais desenvolvidas. No entanto, o excesso de nutrientes pode levar à estabilização e ao declínio do rendimento das culturas (DIAS et al., 2015).

Devido a necessidade de sistemas de cultivos mais sustentáveis, e que ao mesmo tempo, promovam o aumento da produção agrícola, diferentes tecnologias têm sido testadas como alternativas de manejo para substituir o modelo convencional adotado atualmente, principalmente, pelos agricultores familiares (BARBOSA, 2018). Assim sendo, objetivou-se com este trabalho avaliar a produção de frutos de videira Isabel, adubadas com tipos e doses de biofertilizante bovino, durante o segundo ciclo produtivo.

\section{MATERIAL E MÉTODOS}

A pesquisa foi conduzida, no período de fevereiro de 2011 a julho de 2013, e os dados do presente estudo foram obtidos a partir da segunda poda de produção, que ocorreu entre os meses de março a julho de 2013, no Centro de Ciências Humanas e Agrárias - CCHA, da Universidade Estadual da Paraíba - UEPB, no setor de agroecologia, Campus-IV, distando $2 \mathrm{~km}$ da sede do município de Catolé do Rocha, que está situado na região semiárida do estado da Paraíba. A área experimental está localizada nas coordenadas de Latitude $6^{\circ} 20^{\prime} \mathrm{S}$, Longitude $34^{\circ} 44^{\prime}$ e altitude de $275 \mathrm{~m}$ (MASCARENHAS et al., 2006).

Conforme a classificação climática de Köppen-Geiger (ALVARES et al., 2013), o município de Catolé do Rocha possui clima do tipo BSh, ou seja, quente e seco do tipo estepe, com temperatura média anual de $26,9{ }^{\circ} \mathrm{C}$ e evapotranspiração média anual é de $1707,0 \mathrm{~mm}$. A precipitação pluvial média é de $849,1 \mathrm{~mm}$ ao ano, cuja maior parte é concentrada no quadrimestre fevereiro/maio (CEINFO, 2013).

De acordo com o Sistema Brasileiro de Classificação de Solos (EMBRAPA, 2013), o solo da área experimental é classificado como Neossolo Flúvico eutrófico, apresentando a seguinte constituição: $660 \mathrm{~g} \mathrm{~kg}^{-1}$ de areia, 207,6 $\mathrm{g} \mathrm{kg}^{-1}$ de silte e $132,5 \mathrm{~g} \mathrm{~kg}^{-1}$ de argila, com densidade aparente de $1,44 \mathrm{~g} \mathrm{~cm}^{-}$ 3 , umidade de saturação de $234 \mathrm{~g} \mathrm{~kg}^{-1}$, umidade de capacidade de campo de $122,9 \mathrm{~g} \mathrm{~kg}^{-1}$ e umidade de ponto de murcha permanente de $71 \mathrm{~g} \mathrm{~kg}^{-1}$, apresentando $\mathrm{pH}$ de 7,24, CEps de $0,83 \mathrm{dS} \mathrm{m}^{-1}$ e CTC de $5,42 \mathrm{cmol}_{\mathrm{c}} \mathrm{kg}^{-1}$, além de $0,72 \%$ de matéria orgânica. A água utilizada na irrigação apresenta $\mathrm{pH}$ de 7,53, condutividade elétrica de $0,8 \mathrm{dS} \mathrm{m}^{-1}$ e RAS de 2,88 $\left(\mathrm{mmol}_{\mathrm{c}} \mathrm{L}^{-1}\right)^{1 / 2}$.

O delineamento experimental adotado na implantação da pesquisa foi o de blocos casualizados, em esquema fatorial 5 $\mathrm{x} 8$, o primeiro nível referente a cinco tipos de biofertilizantes $\left(\mathrm{B}_{1}=\right.$ à base de esterco bovino, $\mathrm{B}_{2}=$ esterco bovino e farinha de rocha $\mathrm{MB} 4, \mathrm{~B}_{3}=$ esterco bovino, farinha de rocha $\mathrm{MB} 4 \mathrm{e}$ leguminosa (Vigna unguiculata L. Walp.), $\mathrm{B}_{4}=$ esterco bovino, farinha de rocha MB4 e cinza de madeira e $\mathrm{B}_{5}=$ esterco bovino, farinha de rocha MB4, leguminosa e cinza de madeira) e oito doses de biofertilizante $\left(D_{1}=0 ; D_{2}=0,35 ; D_{3}\right.$ $=0,7 ; \mathrm{D}_{4}=1,05 ; \mathrm{D}_{5}=1,4 ; \mathrm{D}_{6}=1,75 ; \mathrm{D}_{7}=2,1 ; \mathrm{e} \quad \mathrm{D}_{8}=2,45$ L/planta/vez), aplicadas a cada 2 meses, no segundo ciclo de produção da videira Isabel, com quatro repetições, totalizando 160 unidades experimentais.

O preparo do solo para o plantio das mudas da videira Isabel constou de uma aração, na profundidade de 0-30 cm e duas gradagens cruzadas. As mudas foram plantadas no espaçamento $3,5 \mathrm{~m}$ x 3,0 m, em covas com dimensões de $50 \mathrm{x}$ 50 x $50 \mathrm{~cm}$, com uma densidade da ordem de 952 plantas por hectare ou 216 plantas na área experimental de 0,22 ha. $\mathrm{O}$ sistema de condução adotado foi em forma de parreira. A adubação de fundação foi feita com esterco bovino curtido, colocando-se $30 \mathrm{~kg} \mathrm{cova}^{-1}$, conforme recomendações da análise de solo. Os atributos químicos da análise do esterco bovino estão apresentados na Tabela 1.

Tabela 1. Atributos químicos do esterco bovino curtido utilizado na adubação de fundação.

\begin{tabular}{ccc}
\hline \multirow{2}{*}{ ESPECIFICAÇÕES } & \multicolumn{2}{c}{ ANÁLISE DO ESTERCO BOVINO } \\
\cline { 2 - 3 } & Obtidos $^{1}$ & Transformados $^{2}$ \\
\hline $\mathrm{pH}$ & 8,10 & - \\
Nutrientes & $(\%)$ & $\left(\mathrm{g} \mathrm{kg}^{-1}\right)$ \\
Nitrogênio & 1,79 & 17,9 \\
Fósforo & 2,08 & 20,8 \\
Potássio & 1,10 & 11,0 \\
Cálcio & 1,68 & 16,8 \\
Magnésio & 0,38 & 3,8 \\
\hline
\end{tabular}

${ }^{1}$ Valores da análise laboratorial, em \%; ${ }^{2}$ Valores transformados, em $\mathrm{g} \mathrm{kg}^{-1}$.

O controle de plantas espontâneas ocorreu sempre que necessário, fazendo-se roços entre as linhas de plantio e capinas manuais nas covas. Foram realizadas podas de formação na videira para definição do número de galhos a 
serem conservados. O controle das doenças fúngicas foi feito de forma preventiva, sendo aplicada a calda bordalesa (à base de sulfato de cobre $250 \mathrm{~g}$, cal hidratada $400 \mathrm{~g}$ e $100 \mathrm{~mL}$ de detergente neutro para $20 \mathrm{~L}$ de água), com o auxílio de um pulverizador costal de $20 \mathrm{~L}$, em intervalo de dez dias. A segunda poda de produção foi realizada em março de 2013, quando as plantas estavam com 25 meses de idade (após o transplantio), deixando-se 40 galhos produtivos por planta, e a colheita foi concluída em julho do mesmo ano.

As adubações de cobertura foram realizadas mensalmente, sendo utilizados os tipos e as doses de biofertilizante para cada tratamento. Os biofertilizantes foram produzidos, de forma anaeróbia, em depósitos (biodigestores) com capacidade individual para $240 \mathrm{~L}$, contendo uma mangueira conectada a um depósito com água, para retirada do gás metano produzido pela fermentação do material. Os biodigestores foram mantidos hermeticamente fechados durante 35 dias, em média, tempo necessário para a conclusão da fermentação do material orgânico pelas bactérias. Em seguida, foram separadas as partes sólida e líquida do biofertilizante, seguindo metodologia proposta por Santos et al. (2014b).

Todos os biofertilizantes foram produzidos a partir de $70 \mathrm{~kg}$ de esterco verde de vacas em lactação e 120 L de água, além de $5 \mathrm{~kg}$ de açúcar e $5 \mathrm{~L}$ de leite para acelerar o metabolismo das bactérias $\left(B_{1}\right)$. Ao $B_{2}$ foi acrescentado $4 \mathrm{~kg}$ de farinha de rocha MB4; para o $\mathrm{B}_{3}$ acrescentou-se $4 \mathrm{~kg}$ de farinha de rocha MB4 e $5 \mathrm{~kg}$ de leguminosa (Vigna unguiculata $\mathrm{L}$. Walp.); no $\mathrm{B}_{4}$ foi adicionado $4 \mathrm{~kg}$ de farinha de rocha $\mathrm{MB} 4$ e $3 \mathrm{~kg}$ de cinza de madeira, e ao $\mathrm{B}_{5}$ foi acrescentado $4 \mathrm{~kg}$ de farinha de rocha MB4, $3 \mathrm{~kg}$ de cinza de madeira e $5 \mathrm{~kg}$ de leguminosa. Após o processo de fermentação, os biofertilizantes foram analisados, e os resultados estão apresentados na Tabela 2. A aplicação dos biofertilizantes foi realizada via solo, sendo aplicada as quantidades de cada tratamento em microbacias circulares, construídas ao redor do caule da planta, realizando-se, em seguida, a irrigação conforme a necessidade diária da cultura.

Tabela 2. Atributos químicos dos biofertilizantes utilizados na pesquisa.

\begin{tabular}{|c|c|c|c|c|c|}
\hline \multirow{2}{*}{ Especificação } & \multicolumn{5}{|c|}{ Tipos de Biofertilizante } \\
\hline & B1 & B2 & B3 & B4 & B5 \\
\hline $\mathrm{pH}$ & 4,68 & 5,15 & 5,94 & 5,09 & 5,25 \\
\hline $\mathrm{CE}\left(\mathrm{dS} \mathrm{m} \mathrm{m}^{-1}\right)$ & 4,70 & 5,70 & 8,54 & 6,81 & 7,10 \\
\hline Fósforo (mg dm ${ }^{-3}$ ) & 296,2 & 338,8 & 388,2 & 394,3 & 403,4 \\
\hline Sódio $\left(\mathrm{cmol}_{\mathrm{c}} \mathrm{dm}^{-3}\right)$ & 1,14 & 0,99 & 1,95 & 1,14 & 1,22 \\
\hline Potássio $\left(\mathrm{cmol}_{\mathrm{c}} \mathrm{dm}^{-3}\right)$ & 0,71 & 0,58 & 0,68 & 1,42 & 1,78 \\
\hline Cálcio $\left(\mathrm{cmol}_{\mathrm{c}} \mathrm{dm}^{-3}\right)$ & 3,75 & 5,75 & 6,00 & 5,10 & 6,00 \\
\hline Magnésio $\left(\mathrm{cmol}_{\mathrm{c}} \mathrm{dm}^{-3}\right)$ & 3,30 & 6,50 & 4,10 & 6,65 & 5,40 \\
\hline Nitrogênio $\left(\mathrm{g} \mathrm{kg}^{-1}\right)$ & 1,00 & 0,80 & 0,80 & 0,70 & 0,80 \\
\hline Enxofre $\left(\mathrm{mg} \mathrm{dm}^{-3}\right)$ & 14,45 & 22,51 & 38,53 & 65,94 & 57,42 \\
\hline
\end{tabular}

A videira foi irrigada através do sistema localizado denominado "Bubller", desenvolvido pela Universidade do Arizona (USA), sendo a água conduzida através de canos e mangueiras utilizando-se a ação da gravidade. As irrigações foram realizadas diariamente, onde as quantidades de água aplicadas foram calculadas com base na evaporação do tanque classe A, repondo-se, no dia seguinte, o volume correspondente à evaporação do dia anterior. $\mathrm{O}$ volume de água diário foi aplicado em microbacias niveladas, construídas ao redor do caule, com diâmetro de 1,0 m, possibilitando uma distribuição uniforme da água no sistema radicular da planta.

Para o cálculo dos volumes de água aplicados, foi considerado o coeficiente do tanque classe A de 0,75 (DOORENBOS; PRUITT, 1977) e os coeficientes de cultivos para os diferentes estádios de desenvolvimento da cultura (DOORENBOS; KASSAN, 1994), além de valores diferenciados de coeficiente de cobertura, ao longo dos ciclos da cultura, sendo a necessidade de irrigação líquida (NIL) diária determinada pela equação (1).

NIL Diária $=0,88 \times$ Kc $x$ Epan $\times$ Cs

Em que, Kc é o coeficiente de cultivo da cultura (tabelado); Epan é a evaporação diária do tanque classe A, em mm; e Cs é o coeficiente de cobertura do solo (tabelado).

A necessidade de irrigação bruta (NIB) foi determinada pela equação (2).

NIB Diária = NIL Diária / (1 - FL) x Ei

Em que: Ei é a eficiência do sistema de irrigação; e FL é a fração de lixiviação, estimada pela equação $\mathrm{FL}=\mathrm{CEa} /(5 \mathrm{x}$ CEes - CEa), onde CEa é a condutividade elétrica da água de irrigação e CEes é a condutividade elétrica limite do extrato de saturação do solo em que o rendimento potencial da cultura ainda é de $100 \%$.

A operação de colheita da uva foi feita quando os cachos atingiam uniformidade de 85 a $90 \%$ do ponto de maturação, sendo acondicionados em embalagens apropriadas. Para a avaliação da produção da videira Isabel foram considerados: o número de bagas por cacho, o peso do cacho, o peso de cachos por planta e o peso da baga, que foram pesadas e determinados em gramas utilizando uma balança comercial. $\mathrm{O}$ teor de sólidos solúveis totais ( ${ }^{\circ}$ Brix) foi determinado por leitura direta em refratômetro, com correção de temperatura, com base em tabela contida no manual do Instituto Adolf Lutz (2005). Na determinação da acidez total titulável, foi utilizado o método acidimétrico da AOAC (1990), por meio de solução padronizada de $\mathrm{NaOH} 0,1 \mathrm{~N}$.

Os efeitos de diferentes tipos e doses de biofertilizante na produção e qualidade da videira Isabel foram avaliados através de análise de variância (teste F), e pela significância dos fatores submeteu-se ao teste de regressão, enquanto o confronto de médias foi feito pelo teste Tukey. Para realização das análises estatísticas, foi utilizado o programa estatístico AGROESTAT (BARBOSA; MALDONADO, 2015).

\section{RESULTADOS E DISCUSSÃO}

Houve diferença significativa $(\mathrm{p}<0,01)$ entre os tipos e doses de biofertilizante em relação a produção de frutos da videira Isabel, sobre o número de bagas por cacho (CV: $12,03 \%$ ), o peso de cachos por planta (CV: $8,97 \%$ ), o peso do cacho (CV: $18,33 \%)$ e o peso da baga (CV: 20,63\%) da videira Isabel na segunda colheita, enquanto que para os atributos qualitativos de ${ }^{\circ}$ Brix (CV: 8,34\%) e acidez (CV: $26,47 \%$ ) não houve diferença significativa em nenhum dos fatores como também na interação. Para os fatores isolados, os tratamentos com tipos e doses causaram efeito em todas as variáveis estudadas ao nível de $1 \%$ de probabilidade, exceto para peso de cachos, peso da baga, ${ }^{\circ}$ Brix e acidez nos tratamentos com doses e tipos, respectivamente, conforme o teste F. Demonstrando que a produção da videira Isabel na $2^{\mathrm{a}}$ colheita é influenciada divergentemente pelas doses e tipos de 
biofertilizantes, mas não interfere em sua qualidade. Fato que pode ser comprovado por Santos et al. (2014b), que não obtiveram resultados significativos dos sólidos solúveis totais e de $\mathrm{pH}$ no fruto da banana nanica ao estudarem diferentes tipos e doses de biofertilizante. As médias de ${ }^{\circ}$ Brix variaram de 18,45 a 19,7 , não tendo havido tendências de aumento e diminuição decorrentes das dosagens de biofertilizante aplicadas. Os valores de acidez total titulável também apresentaram o mesmo comportamento, tendo variado de 3,0 a $3,25 \%$, sem nenhuma supremacia de um tipo sobre o outro.

O desdobramento da interação dose versus tipo de biofertilizante, revelou efeitos significativos das doses dos biofertilizantes $\mathrm{B}_{1}, \mathrm{~B}_{2}, \mathrm{~B}_{4}$ e $\mathrm{B}_{5}$ sobre o número de bagas por cacho da videira Isabel, com aumentos dessa variável ao incrementar a dose de biofertilizante até os limites ótimos de 1,$24 ; 1,27 ; 1,33$ e $1,34 \mathrm{~L} /$ planta/vez, respectivamente, que proporcionaram 23,$2 ; 24,6 ; 25,5$ e 26,2 bagas por cacho, ocorrendo reduções a partir desses patamares (Figura 1), a não significância do tipo $\mathrm{B}_{3}$ pode estar relacionado a alta concentração de sais e $\mathrm{Na}^{+}$em sua composição, que em hipótese, ocorreu devido a ausência de cinza para manter a relação de cargas balanceadas já que possui uma alta concentração de potássio e fósforo (Tabela 2). Esta variação de valores nos tratamentos, provavelmente ocorreu devido a presença de substâncias húmicas contidas no biofertilizante, as quais promovem melhorias no solo, o que consequentemente, favorece a maior absorção de água e nutrientes pelas plantas, estimulando o desenvolvimento e a divisão celular, contribuindo para o aumento de seu desenvolvimento e consequente produção (ARANDA et al., 2015).

Figura 1. Número de bagas por cacho da videira Isabel, na segunda colheita, em função do uso de tipos e doses do biofertilizantes. B1 = à base de esterco bovino, B2 = B1 + farinh de rocha $\mathrm{MB} 4, \mathrm{~B} 4=\mathrm{B} 2+$ cinza de madeira e $\mathrm{B} 5=\mathrm{B} 3+$ cinza d madeira.

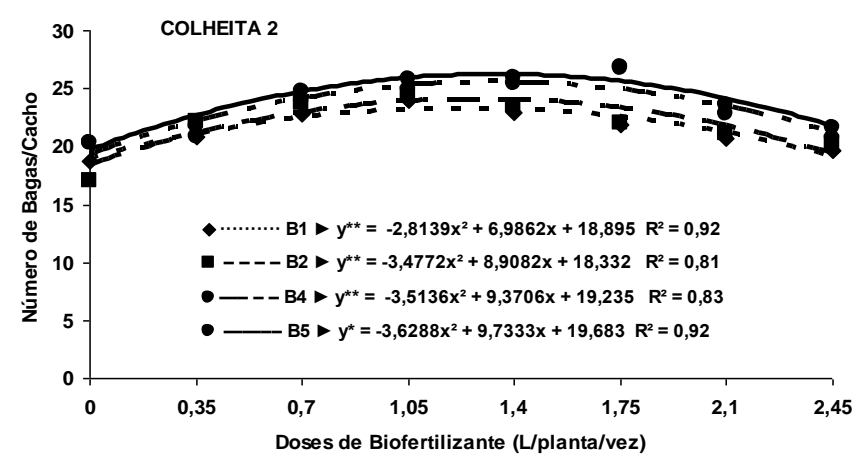

Os aumentos no número de bagas por cacho até as doses ótimas de biofertilizante, possivelmente, ocorreram pelas ações das substâncias húmicas, formadas a partir da aplicação do biofertilizante, as quais podem exercer efeitos nas funções vitais das plantas, resultando, direta ou indiretamente, na absorção de íons e na nutrição mineral das mesmas (SOUZA et al., 2016). Os aumentos verificados também podem ser explicados pela melhoria das características físicas, químicas e biológicas do solo, com o decorrer do tempo (DAMATTO JUNIOR et al., 2009). Nas doses limiares comprova-se que os nutrientes absorvidos pelas plantas durante o crescimento foram utilizados, resultando em maior potencial produtivo, visto que os nutrientes favoreceram as fases de desenvolvimento vegetal e reprodutivo (TAIZ et al., 2017).
As reduções verificadas no número de bagas por cacho nas doses de biofertilizante acima dos limites ótimos podem estar associadas ao aumento do consumo de nutrientes pelos microrganismos do solo, que em condições de elevada fertilidade, se multiplicam de forma intensa, havendo, em consequência disso, diminuição da disponibilidade de nutrientes para as plantas (MALAVOLTA; VITTI; OLIVEIRA, 1997). Observa-se também na Figura 1, que os biofertilizantes com teores mais elevados de nutrientes proporcionaram maiores número de bagas por cacho, para uma mesma dose aplicada, com destaque para o $\mathrm{B}_{5}$ e $\mathrm{B}_{4}$. Segundo Marrocos et al. (2012), a intensidade da melhoria das condições químicas e biológicas do solo depende da riqueza nutricional dos fertilizantes aplicados, uma vez que como em intervalos (15 a 20 dias de decomposição), os biofertilizantes podem acelerar a disponibilidade desses nutrientes às plantas. Assim, esses níveis e intervalos podem ter sido suficientes para nutrir as plantas com os elementos essenciais, e acima dessas doses, podem ter apresentado efeito deletério. Provavelmente, durante o desenvolvimento das plantas, as doses de biofertilizante bovino, juntamente com os nutrientes contidos nos substratos, podem ter suprido eficientemente as necessidades nutricionais da videira Isabel, principalmente na sua fase reprodutiva.

O desdobramento da interação dose versus tipo de biofertilizante revelou efeitos significativos de doses dos tipos $\mathrm{B}_{1}, \mathrm{~B}_{2}$ e $\mathrm{B}_{5}$ sobre o peso de cachos por planta, observando aumentos dessa variável com o incremento da dose de biofertilizante até os limites ótimos de 1,31; 1,34 e 1,40 L/planta/vez, respectivamente, que proporcionaram 4,8; 5 e $5,3 \mathrm{~kg}$ de cachos por planta, constatando reduções a partir desses limites (Figura 2). Observa-se que o biofertilizante $\mathrm{B}_{5}$ proporcionou um peso de cachos por planta superior aos obtidos nos tipos $B_{1}$ e $B_{2}$, para uma mesma dose aplicada, o gue pode ser explicado hipoteticamente devido a maior quantidade de ingredientes ricos em elementos químicos, que proporcionou uma disponibilidade de nutrientes para as plantas. Com relação aos valores eficientes obtidos neste experimento, constatou-se que o biofertilizante pode ser uma alternativa viável para o produtor, em consenso com Sá et al. (2013), ao afirmarem que a substituição parcial de fertilizantes minerais pelos biofertilizantes em solução nutritiva para o cultivo do mamoeiro, resulta em produtividades adequadas, semelhante ao ocorrido na videira Isabel para o peso de cachos por planta.

Figura 2. Peso de cachos por planta da videira Isabel, na segunda colheita, em função do uso de tipos e doses dos biofertilizantes. $\mathrm{B} 1=$ à base de esterco bovino, $\mathrm{B} 2=\mathrm{B} 1+$ farinha de rocha MB4 e B5 = B2 + leguminosa + cinza de madeira.

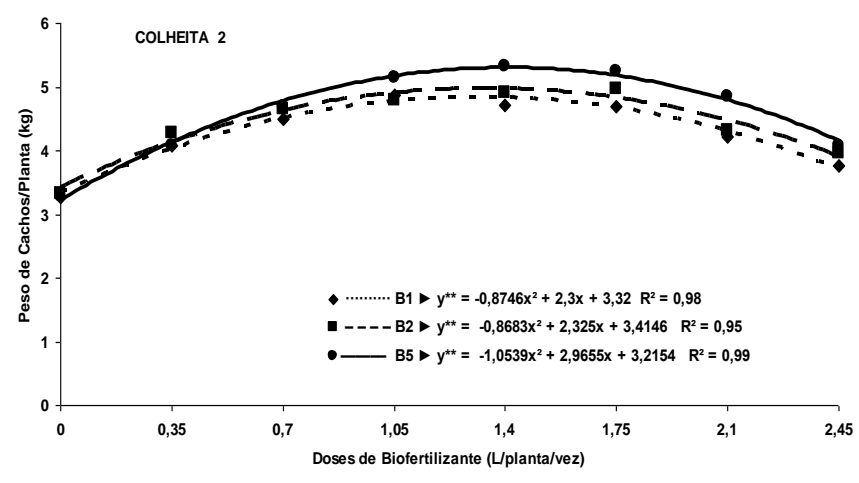


Os aumentos verificados no peso de cachos por planta até as doses ótimas de biofertilizante podem ser atribuídos à oferta crescente de nutrientes para as plantas via solo, resultando em uma nutrição mais equilibrada (BLOOM et al., 2014). Os aumentos verificados também podem ser explicados pela elevação na quantidade de nutrientes disponíveis às plantas, principalmente o nitrogênio, que, segundo Epstein e Bloom (2006), é um elemento com função estrutural na planta, fazendo parte de moléculas de aminoácidos e proteínas, além de ser constituinte de bases nitrogenadas e ácidos nucléicos. Este efeito positivo do biofertilizante pode estar relacionado ao efeito da matéria orgânica, proporcionando resultados positivos diretos no solo, como diminuição da compactação, aumento da retenção de água e melhor disponibilidade de nutrientes (CONDÉ et al., 2017).

As reduções ocorridas no peso de cachos por planta nas doses de biofertilizante superiores aos limites ótimos, também podem estar associadas ao excesso de nutrientes fornecidos nas adubações, causando fitotoxicidade às plantas (INSELSBACHER et al., 2013). O excesso de potássio na planta pode provocar efeitos adversos de toxicidade, diminuindo o crescimento e a produção das plantas (FARIA et al., 2012; VIEIRA et al., 2016), reduzindo o peso de cachos por planta, em consequência desse elemento desempenhar papel importante em processos osmóticos, na expansão celular, na fotossíntese, na permeabilidade das membranas, no controle do $\mathrm{pH}$, no transporte de açúcares pelo floema e em mecanismos de defesa das plantas contra pragas e doenças.

O desdobramento da interação dose versus tipo de biofertilizante revelou efeitos significativos de doses dos biofertilizantes $\mathrm{B}_{1}, \mathrm{~B}_{2}, \mathrm{~B}_{4}$ e $\mathrm{B}_{5}$ sobre o peso do cacho, ocorrendo aumentos dessa variável com o incremento das doses de biofertilizantes até os limites ótimos de 1,48; 1,45; 1,47 e $1,48 \mathrm{~L} /$ planta/vez, respectivamente, que proporcionaram a produção de cachos com 84,2; 87,6; 95,1 e 96,6 g, respectivamente, havendo reduções a partir desses limites (Figura 3). Observa-se que, para uma mesma dose aplicada, os biofertilizantes $\mathrm{B}_{4}$ e $\mathrm{B}_{5}$, por conterem teores maiores de nutrientes, proporcionaram valores de peso do cacho superiores aos dos tipos $\mathrm{B}_{1}$ e $\mathrm{B}_{2}$. $\mathrm{O}$ tamanho do cacho, proporcionado pela dose ótima $(1,48 \mathrm{~L} /$ planta/vez $)$ do biofertilizante $\mathrm{B}_{5}$, pode ser observado na Figura 3 .

Figura 3. Peso do cacho da videira Isabel, na segunda colheita, em função do uso de diferentes doses de biofertilizante. $\mathrm{B} 1=$ à base de esterco bovino, $\mathrm{B} 2=\mathrm{B} 1+$ farinha de rocha $\mathrm{MB} 4, \mathrm{~B} 4=\mathrm{B} 2+$ cinza de madeira e B5 = B3 + leguminosa + cinza de madeira.

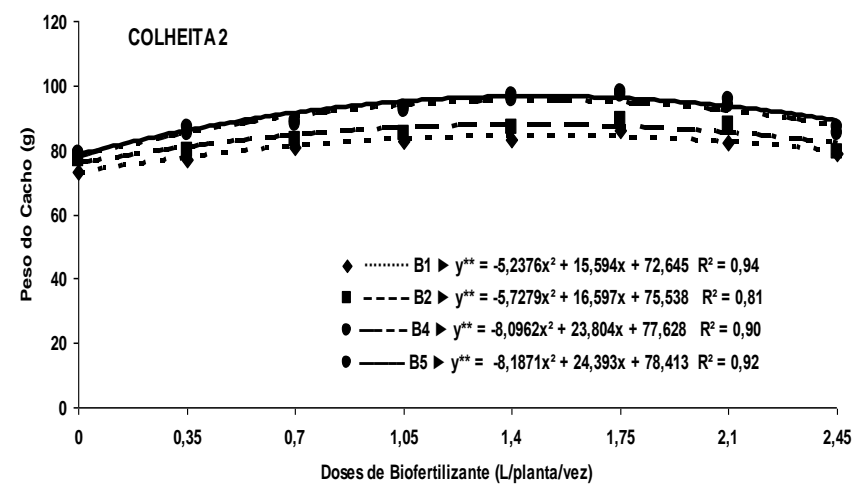

Os resultados encontrados neste experimento evidenciaram que, quando utilizado como fonte orgânica, o biofertilizante bovino, atenua o efeito da ausência de nutrientes no solo, onde as plantas foram cultivadas. Vale ressaltar que além de frutíferas, o efeito positivo promovido pelo biofertilizante bovino sobre o rendimento, já foi verificado em hortaliças tipo fruto como o melão (SANTOS et al., 2014a).

As razões dos aumentos do peso do cacho até as doses ótimas de biofertilizante são fundamentadas nas teorias defendidas por Dosani et al. (1999) e Epstein e Bloom (2006), mencionadas para o peso de cachos por planta. $\mathrm{O}$ fornecimento de nitrogênio, fósforo e potássio, entre outros elementos essenciais às plantas, pelos biofertilizantes é de fundamental importância para as funções fisiológicas das mesmas, especialmente o nitrogênio, que é constituinte de todos os aminoácidos, proteínas e nucleotídeos (FERNANDES et al., 2018). Os motivos das reduções ocorridas no peso do cacho quando foram aplicadas doses de biofertilizante superiores aos limites ótimos, são os mesmos apontados para o peso de cachos por planta.

O desdobramento da interação dose versus tipo de biofertilizante revelou efeitos significativos de doses dos tipos $\mathrm{B}_{1}, \mathrm{~B}_{2}, \mathrm{~B}_{4}$ e $\mathrm{B}_{5}$ sobre o peso da baga da videira Isabel, promovendo aumentos dessa variável com o incremento da dose de biofertilizantes até os limites ótimos de 0,95;0,96; 1,02 e $1,08 \mathrm{~L} / \mathrm{planta} / \mathrm{vez}$, respectivamente, que proporcionaram a produção de bagas com 4,2; 4,3; 4,4 e 4,5 $\mathrm{g}$, havendo reduções a partir desses patamares (Figura 4). As razões dos aumentos e das reduções são as mesmas apontadas para o peso do cacho. Observa-se que os biofertilizantes mais ricos em nutrientes proporcionaram valores de peso da baga superiores aos mais pobres $\left(\mathrm{B}_{5}>\mathrm{B}_{4}>\mathrm{B}_{2}>\mathrm{B}_{1}\right)$, para uma mesma dose aplicada. Vale salientar que, o biofertilizante tem demonstrado eficiência em várias frutíferas como no maracujazeiro amarelo (NUNES et al., 2016), mamoeiro (LIMA NETO et al., 2016) e goiabeira (ROCHA et al., 2016). Santos et al. (2014b) estudando o efeito de doses de biofertilizante em bananeira nanica também observou eficiência com diferentes tipos, em que o biofertilizante com maior composição de ingredientes afetou consideravelmente a produção de frutos.

Figura 4. Peso da baga da videira Isabel, na segunda colheita, em função do uso de diferentes doses de biofertilizante. B1 = à base de esterco bovino, $\mathrm{B} 2=\mathrm{B} 1+$ farinha de rocha $\mathrm{MB} 4$, $\mathrm{B} 4=\mathrm{B} 2+$ cinza de madeira e B5 = B3 + leguminosa + cinza de madeira.

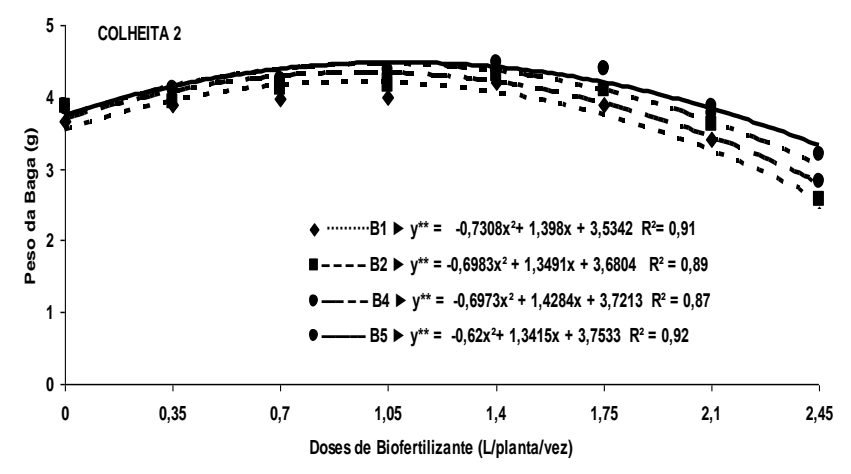




\section{CONCLUSÕES}

O uso de biofertilizante de esterco bovino enriquecido com farinha de rocha MB4, cinza de madeira e leguminosa (Vigna unguiculata L. Walp.) na dose 1,2 L/planta/vez aplicado mensalmente proporciona maior eficiência produtiva de frutos de videira Isabel.

A aplicação dos biofertilizantes enriquecidos com dosagens adequadas acarreta em plantas bem desenvolvidas nutricionalmente, podendo, desta forma, ser recomendado na fertilização da videira Isabel.

\section{REFERÊNCIAS}

ALVARES, C. A.; STAPE, J. L.; SENTELHAS, P. C.; GONÇALVES, J. L. M.; SPAROVEK, G. Köppen's climate classification map for Brazil. Meteorologische Zeitschrift, v.22, p.711-728, 2013. https://doi.org/10.1127/0941$\underline{2948 / 2013 / 0507}$

AOAC, Association of Official Analytical Chemists. Official Methods of Analysis. 15th ed. v.1, Arlington: AOAC, 1990.p.768.

https://law.resource.org/pub/us/cfr/ibr/002/aoac.methods.1.19 90.pdf.

ARANDA, V.; MACCI, C.; PERUZZI, E.; MASCIANDARO, G. Biochemical activity and chemicalstructural properties of soil organic matter after 17 years of amendments with olivemill pomace co-compost. Journal of Environmental Management, v.147, p.278-285, 2015 https://doi.org/10.1016/j.jenvman.2014.08.024.

BARBOSA, J. C.; MALDONADO, JUNIOR, W. AgroEstat sistema para análises estatísticas de ensaios agronômicos. Jaboticabal: FCAV/UNESP, 2015. 396 p.

BARBOSA, S. J. C. Utilização de Biofertilizante Bovino liquido em cultivo de alface crespa (cv. Vanda). 2018. $64 \mathrm{f}$ Dissertação (Mestrado em Agroecologia e Desenvolvimento Rural) - Universidade Federal de São Carlos, Araras, 2018.

BARROSO NETO, J.; SOUSA, I. F. Potencial climático para cultivo da videira no alto sertão sergipano. Revista Brasileira de Agricultura Irrigada, v.12, n.5, p.2932-2943, 2018.

BLOOM, A. J.; BURGER, M.; KIMBALL, B. A.; PINTER, P. J. Nitrate assimilation is inhibited by elevated $\mathrm{CO} 2$ in field-grown wheat. Nat clim change, v.4, n.6, p.477-80, 2014. https://doi.org/10.1038/nclimate2183

CEINFO. Centro de informações tecnológicas e comerciais para fruticultura tropical. Banco de dados pluviométricos e pedológicos do Nordeste. Disponível em: http://www.ceinfo.cnpat.embrapa.br. Acesso em: 10 jan. 2018.

CHICONATO, D. A.; SIMONI, F.; GALBIATTI, J. A.; FRANCO, C. F.; CARAMELO, A. D. Resposta da alface à aplicação de biofertilizante sob dois níveis de irrigação. Bioscience Journal, v. 29, n. 2, p.392-399, 2013.
CONDÉ, V. F.; OLIVEIRA, D. M. F.; OLIVEIRA, J. E. Z. Incidência e severidade de hérnia das crucíferas em repolho (Brassica oleracea L. var. capitata) em solo tratado com biofertilizante tipo Bokashi. Ciência e Natura, v.39, n.1, p.0715, 2017. https://doi.org/10.5902/2179460X21445.

DAMATTO JUNIOR, E. R.; NOMURA, E. S.; FUZITANI, E. J.; SAES, L. A. Experiências com o uso de adubação orgânica na cultura da banana. In: GODOY, L. J. G.; GOMES, J. M. Tópicos sobre nutrição e adubação da cultura da banana. Botucatu: FEPAF/UNESP, 2009. p.94-120.

DOORENBOS, J.; KASSAM, A.H.; Efeito da água no rendimento das culturas. Tradução de GHEYI, H. R.; SOUSA, A. A.; DAMASCENO, F. A. V.; MEDEIROS, J. F. (Estudos FAO: Irrigação e Drenagem, 33), Campina Grande: Universidade Federal da Paraíba - UFPB, 1994. 306p.

DIAS, C. N.; MARINHO, A. B.; ARRUDA, R. S.; SILVA, J. P.; PEREIRA, E. D.; FERNANDES, C. N. V. Produtividade e qualidade do morangueiro sob dois ambientes e doses de biofertilizante. Revista Brasileira de Engenharia Agrícola e Ambiental, v. 19, n. 10, p.961-966, 2015. https://doi.org/10.1590/1807-1929.

DOORENBOS, J.; PRUITT, W. O. Guidelines for predicting crop water water requirements. (FAO Irrigation and Drainage Paper 24), 2 ed. Rome: FAO, 1977. 156p.

DOSANI, A. A. K.; TALASHILKAR, S. C; MEHTA, V. B. Effect of poultry manure applied in combination with fertilizers on the yield, quality and nutrient uptake of groundnut. J. Indian Soc. Soil Sci., v.47 , p.166-169, 1999.

EMBRAPA, Empresa Brasileira de Pesquisa Agropecuária. Sistema Brasileiro de Classificação de Solos. 4. Ed., Brasília: Embrapa Solos, 2013. 253p.

EPSTEIN, E.; BLOOM, A. J. Nutrição mineral de plantas: princípios e perspectivas. Tradução de Nunes, M. E. T. 2. ed. Londrina: Editora Planta, 2006. 401p.

FARIA, A. F.; ALVAREZ, V. V. H.; MATTIELLO E. M.; LIMA, N. J. C.; FELIX, B. N.; NOGUEIRA, P. H. Capacidade de suprimento de potássio em solos de Minas Gerais - Brasil. Spanish Journal of Soil Sciense, v.22, n.1, p.26-37, 2012. https://doi.org/10.3232/SJSS.2012.V2.N1.02.

FERNANDES, M. S.; SOUZA, S. R.; SANTOS, L. A. Nutrição mineral de plantas. $2^{\mathrm{a}}$ ed. Viçosa, MG: Sociedade Brasileira de Ciência do Solo, 2018. 670p.

FERRANTI, T. H. Caracterização de compostos fenólicos de sucos de Vitis labrusca variedade Bordô sob diferentes sistemas de manejo agrícola. 2017. 44f. Dissertação (Mestrado em Biotecnologia), Universidade de Caxias do Sul, 2017.

INSELSBACHER, E.; WANEK， W.; STRAUSS， J.; ZECHMEISTER-BOLTENSTERN, S.; MÜLLER, C. A novel $15 \mathrm{~N}$ tracer model reveals: Plant nitrate uptake governs nitrogen transformation rates in agricultural soils. Soil Biology and Biochemistry, v.57, p.301-310, 2013. https://doi.org/10.1016/j.soilbio.2012.10.010. 
INSTITUTO ADOLFO LUTZ. Normas analíticas do Instituto Adolfo Lutz: métodos químicos e físicos para análise de alimentos. 3.ed. São Paulo: 2005. 533p.

KIST, B. B. et al. Anuário brasileiro da fruticultura 2018. Santa Cruz do Sul: Editora Gazeta Santa Cruz, 2018.

LIMA, A. S.; ALVES, J. M.; MESQUITA, F. O.; MESQUITA, E. F.; SOUSA, C. S.; SILVA, F. L.; ALVES, A. S.; SOARES, L. S. Organic Fertilization and Hydric Reposition in the Initial Production of Passiflora edullis. $f$. flavicarca Deg. Journal of Experimental Agriculture International, v.30, p.01-14, 2019.

LIMA NETO, A. J.; CAVALCANTE, L. F.; MESQUITA, F. O.; SOUTO, A. G. L.; SANTOS, G. P.; SANTOS, J. Z.; MESQUITA, E. F. Papaya seedlings irrigation with saline water in soil with bovine biofertilizer. Chilean Journal of Agricultural Research, v. 76, p.235-241, 2016. https://doi.org/10.4067/S0718-58392016000200014.

MALAVOLTA, E.; VITTI, G. C.; OLIVEIRA, S. A. Avaliação do estado nutricional das plantas: princípios e aplicações. Piracicaba: POTAFOS, 2. ed., 1997. 201p.

MAPA. Ministério da Agricultura, Pecuária e Abastecimento. Uva. Disponível em: <http://www.agricultura.gov.br/assuntos/politicaagricola/todas-publicacoes-de-politica-agricola/projecoes-doagronegocio/proj_agronegocio2016.pdf/@ @download/file/Pr oj_Agronegocio2016.pdf>. Acesso em 23 outubro de 2015.

MARROCOS, S. T. P.; JUNIOR, J. N.; GRANGEIRO, L. C.; AMBROSIO, M. M. Q.; CUNHA, A. P. A. Composição química e microbiológica de biofertilizantes em diferentes tempos de decomposição. Revista Caatinga, Mossoró, v. 25 n. 4, p.34-43, 2012.

MASCARENHAS, J. C.; BELTRÃO, B. A.; JUNIOR, L. C. S.; MORAIS, F.; MENDES, V. A.; MIRANDA, J. L. F. Serviço Geológico do Brasil: Diagnóstico do município de Catolé do Rocha. Recife: CPRM/PRODEEM, 2005. 21p.

MAZARO, S. M.; MANGNABOSCO, M. C.; CITADIN, I.; PAULUS, D.; GOUVEA, A. Produção e qualidade de morangueiro sob diferentes concentrações de calda bordalesa, sulfocálcica e biofertilizante supermagro. Semina: Ciências Agrárias, v. 34, n. 6, p.3285-3294, 2013. https://doi.org/10.5433/1679-0359.2013v34n6Supl1p3285.

MELLO, L. M. R. Relatório da avaliação de impactos da uva Niagara Rosada para regiões tropicais. Embrapa, Bento Gonçalves, 2016. Disponível em: <https://bs.sede.embrapa.br/2016/relatorios/uvaevinho_2016_ uvasemsemente.pdf>. Acesso em: 20 jul. 2017.

NUNES, J. C.; CAVALCANTE, L. F.; PEREIRA, W. E.; NETO, J. A. L ; SILVA, J. A. ; OLIVEIRA, F. F . Seedlings of yellow passion fruit in soils degraded by salts treated with bovine biofertilizer. Científica, v.44, n.1, p.91-91, 2016. https://doi.org/10.15361/1984-5529.2016v44n1p91-101
OLIVEIRA, F. I. F.; MEDEIROS, W. J. F.; CAVALCANTE, L. F.; CAVALCANTE, Í. H. L.; SOUTO, A. G. L.; NETO, A. J. L. Crescimento e produção do maracujazeiro amarelo fertirrigado com esterco bovino líquido fermentado. Revista Agropecuária Técnica, v.38, n.4, p.191-199, 2017. https://doi.org/10.25066/agrotec.v38i4.34434

ROCHA, L. F.; CAVALCANTE, L. F.; NUNES, J. C.; SOUTO, A. G. L; CAVALCANTE, A. C. P.; CAVALCANTE, I. H. L.; PEREIRA, W. E. Fruit production and quality of guava Paluma as a function of humic substances and soil mulching. African Journal of Biotechnology, v. 15, n.36, p. 1962-1969, 2016. https://doi.org/10.5897/AJB2016.15587.

SÁ, F. V. S.; MESQUITA, E. F.; BERTINO, A. M. P.; SILVA, G. A.; COSTA, J. D. Biofertilizantes na produção hidropônica de mudas de mamoeiro. Revista Verde de Agroecologia e Desenvolvimento Sustentável, v.8, n.3, p.109116, 2013.

SANTOS, A. P. G.; VIANA, T. V. A.; SOUSA, G. G.; Ó, L. M. G.; AZEVEDO, B. M.; SANTOS, A. M. Produtividade e qualidade de frutos do meloeiro em função de tipos e doses de biofertilizantes. Horticultura Brasileira, v.32, n.4, p.409-416, 2014. https://doi.org/10.1590/S0102-053620140000400007.

SANTOS, J. G. R.; ANDRADE, R.; GALDINO, P. O.; LINHARES, A. S. F.; MAIA, P. M. E.; LIMA, A. S. Qualidade da produção da bananeira Nanicão em função do uso de biofertilizantes. Revista Brasileira de Engenharia Agrícola e Ambiental, v.18, n.4, p.387-393, 2014. https://doi.org/10.1590/S1415-43662014000400005.

SOUZA, J. T. A.; CAVALCANTE, L. F.; NUNES, J. C.; BEZERRA, F. T. C.; NUNES, J. A. S.; SILVA, A. R.; ORESCA, D.; CAVALCANTE, A. G. Effect of saline water, bovine biofertilizer and potassium on yellow passion fruit growth after planting and on soil salinity. African Journal of Agricultural Research, v.11, n.32, p.2994-3003, 2016. https://doi.org/10.5897/AJAR2016.11233

TAIZ, L.; ZEIGER, E.; MOLLER, I. M.; MURPHY, A. Fisiologia e desenvolvimento vegetal. Porto Alegre: Artmed, 6 ed, 2017. p. 858.

VIEIRA, M. S.; OLIVEIRA, F. H. T.; SANTOS, H. C.; MEDEIROS, J. S. Contribution of non-exchangeable potassium forms and its accumulation in corn plants. Revista Brasileira de Engenharia Agrícola e Ambiental, v.20, n.1, p.915, 2016. https://doi.org/10.1590/1807-1929/agriambi. v20n1p9-15. 